\section{Dr. Lequerré, et al reply}

\section{To the Editor:}

We thank Dr. Kapoor for his useful and expert opinion on our recent arti$\mathrm{cle}^{1}$. He reports the rapidly expanding role and benefits of anakinra in the management of nonarthritic systemic disorders. Indeed, anakinra decreases necrosis in acute myocardial infarctions, reduces pulmonary inflammation and hypertension, and prevents $\beta$-cell apoptosis in diabetes.

Initially, anakinra was developed only for treating rheumatoid arthritis (RA), and several studies showed that recombinant interleukin 1 (IL-1) receptor antagonist attenuated RA symptoms and signs, and slowed bone and cartilage destruction. Recently, it was shown that anakinra in combination with disease modifying antirheumatic drugs improves functional status in patients with active disease ${ }^{1}$. However, even if a subset of patients might be particularly responsive to the anakinra-methotrexate combination, this drug is today prescribed less often because it is considered less active on disease activity than anti-tumor necrosis factor agents or other immunotherapies such as anti-CD20 (rituximab) or CTLA4-Ig (abatacept $)^{2}$. However, there has been no head-to-head comparison of anakinra with other biologics. Moreover, anakinra was also shown to be very effective in adult-onset Still's disease (AOSD), systemic-onset juvenile idiopathic arthritis (SOJIA) ${ }^{3}$, Schnitzler syndrome, and autoinflammatory diseases such as the hyperimmunoglobulinemia $\mathrm{D}$ with periodic fever syndrome (HIDS), the cryopyrin-associated periodic syndromes including Muckle-Wells syndrome (MWS), chronic infantile neurologic, cutaneous, articular (CINCA) syndrome, and familial cold urticaria ${ }^{4}$. In these diseases, anakinra improves joint inflammation and also systemic manifestations, as precisely reported by Dr. Kapoor. Indeed, in several patients with HIDS, CINCA, and MWS, rapid and sustained benefits were obtained, often with resolution of rash, fever, asthenia, and abdominal pain. In addition, improvements in symptoms such as hydrocephalus in a patient with CINCA and hearing loss in a patient with MWS were reported ${ }^{4}$. In several patients with Schnitzler syndrome, anakinra was also markedly effective for monoclonal gammopathy, urticaria, intermittent fever, arthralgia and/or arthritis, liver and spleen enlargement, and lymphadenopathy ${ }^{5}$. In a retrospective study, we assessed the efficacy of anakinra treatment in SOJIA and $\mathrm{AOSD}^{3}$.

In our study ${ }^{3}, 15$ of 20 patients with SOJIA treated with anakinra showed some improvement. Clinical systemic features, including fever and rash, were resolved in 14 cases within the first 3 months. Eleven of 15 (73\%) patients with AOSD had a prompt and dramatic improvement in all disease symptoms and signs despite the severity of their disease. All clinical and biological markers in 9 of the $15(60 \%)$ patients improved by at least $50 \%$ at 6 months. In our study, neutralization of IL-1 might be more effective in patients with highly active systemic disease than in patients with chronic arthritis with fewer or no systemic symptoms ${ }^{3}$. These observations confirm the sustained efficacy of anakinra on systemic manifesta- tions. They also emphasize that IL-1ß is involved in the pathophysiology of many diseases, and surprisingly, in some unexpected pathologies.

IL-1B is a useful therapeutic target in several diseases with systemic disorders. In RA, the moderate efficacy of anakinra could be explained at least by its limited bioavailability over the 24 -hour cycle ${ }^{2}$. Moreover, this relative efficacy may reflect the variation of cytokine patterns among patients, and over time in an individual patient with RA. IL-1 inhibition is probably also effective only within a limited "window" during the pathophysiological process ${ }^{6}$. In many systemic diseases, anakinra clearly improves clinical manifestations such as fever, cutaneous rash, sensorineural deafness, abdominal pain, and lymphadenopathy. As suggested by Dr. Kapoor, it is critical to break up the diseases with an IL- $1 \beta$ signature with genomic approaches such as transcriptomic tools.

THIERRY LEQUERRÉ, MD, PhD; OLIVIER VITTECOQ, MD, PhD; XAVIER LE LOËT, MD, Department of Rheumatology, Rouen University Hospital, INSERM 905 and IFRMP23, Institute for Biomedical Research, University of Rouen, 76031 Rouen Cedex, France. Address reprint requests to Dr. Le Loët; E-mail: xavier.le-loet@chu-rouen.fr

\section{REFERENCES}

1. Le Loet X, Nordstrom D, Rodriguez M, et al. Effect of anakinra on functional status in patients with active rheumatoid arthritis receiving concomitant therapy with traditional disease modifying antirheumatic drugs: Evidence from the OMEGA Trial. J Rheumatol 2008;35:1538-44.

2. Burger D, Dayer JM, Palmer G, Gabay C. Is IL-1 a good therapeutic target in the treatment of arthritis? Best Pract Res Clin Rheumatol 2006;20:879-96.

3. Lequerré T, Quartier P, Rosellini D, et al; Société Francophone pour la Rhumatologie et les Maladies Inflammatoires en Pédiatrie (SOFREMIP); Club Rhumatismes et Inflammation (CRI). Interleukin-1 receptor antagonist (anakinra) treatment in patients with systemic-onset juvenile idiopathic arthritis or adult onset Still disease: preliminary experience in France. Ann Rheum Dis 2008;67:302-8.

4. Lequerré T, Vittecoq O, Le Loët X. What is the role for interleukin1 receptor antagonist in rheumatic disease? Joint Bone Spine 2007;74:223-6.

5. de Koning HD, Bodar EJ, Simon A, van der Hilst JC, Netea MG, van der Meer JW. Beneficial response to anakinra and thalidomide in Schnitzler's syndrome. Ann Rheum Dis 2006;65:542-4.

6. Dayer JM. The saga of the discovery of IL-1 and TNF and their specific inhibitors in the pathogenesis and treatment of rheumatoid arthritis. Joint Bone Spine 2002;69:123-32.

J Rheumatol 2009;36:2; doi:10.3899/jrheum.081043 